fit with an unusual pattern or rate of spread of electrical activity within the cortex.

A. JAMES
D. SIMPSON
Adolescent Unit, The Bethlem Royal Hospital, Monks
Orchard Road, Beckenham, Kent BR3 3BX

References

Christensen, Peder \& Koldbaek. IB. (1982) EEG monitored ECT. British Journal of Psychiatry, 141, 19-23.

Blumenthal. I. J. (1955) Spontaneous seizure and related encephalographic findings following shock therapy. Journal of Nervous and Mental Diseases, 122, 581-8.

\section{RATING TARDIVE DYSKINESIA}

DeAR SiR,

The paper by Bergen et al (Journal, May 1984, 144, 498-502) found marked intra-rater variability in the assessment of tardive dyskinesia in four patients, using the Abnormal Involuntary Movements Scale (AIMS). This finding casts further doubt on the usefulness of this, and similar scales, for the measurement of tardive dyskinesia. the AIMS was originally developed to quantify the severity of spontaneous, involuntary movements, and consists of a check-list of movements at various body sites. Brief descriptions of the movements to be rated are provided, but these are too limited to allow clear distinction between abnormal (dyskinetic) movements and normal, fidgety movements, the motor phenomena of akathisia, tics, and schizophrenic stereotypies and mannerisms. No criteria for severity are provided, and, as Bergen et al comment, the rater arrives at a global judgement of severity based on the character, amplitude and frequency of a movement. Although satisfactory interrater reliability has been demonstrated (Smith et al, $1979 \mathrm{a}, \mathrm{b})$, whether AIMS scores are a valid reflection of tardive dyskinesia has not been demonstrated. In research, the use of the scale as a diagnostic instrument, with arbitrary cutoff scores, can yield a patient sample that is markedly heterogeneous with regard to type and distribution of movements.

Bergen and his colleagues stated that they were not aware of any other study which had presented re-rating data. This may be true for the AIMS, but my own study (Barnes \& Trauer, 1982) analysed ratings on 94 patients, by six raters using a videotape assessment procedure, and found high inter-rater and re-rating reliability. Like the AIMS, this scale involves the rating of movement at a number of body sites, and it suffers from some of the same drawbacks. However, the measure of severity was specified as the proportion of time a movement was present while the patient was being observed, a criterion that seemed a reasonable reflection of clinical morbidity. Validity was tested by comparison of the ratings with the diagnostic opinion of independent, experienced clinicians. Ratings for oro-facial movements showed close agreement with the clinicians' evaluation of the severity of tardive dyskinesia, and a diagnostic threshold score was set for ratings of oro-facial movement. Ratings for trunk and limb movements did not show good agreement with the clinicians' diagnostic assessment. For such movements, simple, quantitative measures of frequency, amplitude or duration of movement are inadequate, as they fail to discriminate between tardive dyskinesia of the trunk and limbs and the variety of other movements of the extremities that may be present in psychotic patients on long-term treatment with antipsychotic drugs.

\section{St. George's Hospital Medical School, Cranmer Terrace, Tooting SW17 ORE}

Thomas R. E. BARNeS

\section{References}

Smith, J. M., Kucharskı, L. T., Oswald, W. T. \& Waterman, L. J. (1979a) A systematic investigation of tardive dyskinesia in inpatients. American Journal of Psychiatry, 136, 918-22.

Eblen, C., Knutsen, E. \& Linn, C. (1979b) An assessment of tardive dyskinesia in schizophrenic out-patients. Psychopharmacology, 64, 99-104.

Barnes, T. R. E. \& Trauer, T. (1982) Reliability and validity of a tardive dyskinesia videotape rating technique. British Journal of Psychiatry, 140, 508-15.

DEAR SIR,

\section{IN THE BEGINNING . . .}

I was interested to read Philippe Ployé's article (Journal, July 1984, 145, 55-8) on the possible antecedents of projective identification and idealisation in the last three months of pregnancy.

Why three months? Are not the basic elements of the personality present from conception? The pointed, motile, energetic sperm and the round, passive, receptive egg form the creative zygote which has a desire to reproduce itself, to put out, to take in, a need to relate and a drive to grow, to mature and become a person.

May I ask another question? What might be the psychological sequelae of freezing embryos? Would we predict a deep seated fear of stagnant dependent immortality, alongside the fear of death?

Geoff LAYToN

John Connolly Hospital, Birmingham B45

\section{CORRECTION}

Please note the following is the correct key to Figure 3 in the paper by David Sturgeon et al published in the Journal, July 1984, 145, 62-9.

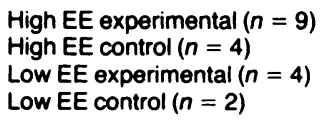

